\title{
Factors Associated with In-hospital Mortality in Severe Burn Patients in Songklanagarind Hospital: A Retrospective Study
}

\author{
Atthawit Mongkornwong, M.D. ${ }^{1}$, Rassamee Sangthong, M.D., Ph.D. ${ }^{2}$, \\ Thara Tunthanathip, M.D., Ph.D. ${ }^{1}$ Surasak Sangkhathat, M.D., Ph.D. ${ }^{1}$
}

${ }^{1}$ Division of Plastic and Reconstructive Surgery, Department of Surgery, Faculty of Medicine, Prince of Songkla University, Hat Yai, Songkhla 90110, Thailand.

${ }^{2}$ Department of Epidemiology, Faculty of Medicine, Prince of Songkla University, Hat Yai, Songkhla 90110, Thailand.

Received 3 February 2020 • Revised 17 July 2020 • Accepted 30 July 2020 • Published online 18 November 2020

\begin{abstract}
:
Objective: This study aimed to analyze the factors associated with mortality in burn patients in order to develop a nomogram for predicting mortality that can be used as a guideline to treat major burn patients.

Material and Methods: This was a retrospective study done in Songhklanagarind Hospital, Hat Yai, Songkhla, Thailand. The study included major burn patients who were admitted to the burn unit or the intensive care unit between the years 2011 and 2018, and analyzed demographic data and significant factors associated with mortality.

Results: A total of 127 major burn patients were treated during the study years, with a mean age of 33 years with an average total burn surface area (TBSA) of $43.5 \%$. The most common cause of the burns was flame injury, and the most significant factors associated with mortality were TBSA $>55.0 \%$, blood creatinine $>1.2$ milligrams per deciliter (mg/dL) and face involvement.
\end{abstract}

Conclusion: The 3 significant burn-related factors of TBSA $>55.0 \%$, blood creatinine $>1.2 \mathrm{mg} / \mathrm{dL}$ and face involvement can be used to predict mortality using the nomogram created in this study.

Keywords: low-to-middle-income countries, major burn patients, mortality, nomogram

Contact: Atthawit Mongkornwong, M.D.

Division of Plastic and Reconstructive Surgery, Department of Surgery, Faculty of Medicine,

Prince of Songkla University, Hat Yai, Songkhla 90110, Thailand.

E-mail: Atthawit_sx@hotmail.com

政

This is an open access article under the CC BY-NC-ND license

(http://www.jhsmr.org/index.php/jhsmr/about/editorialPolicies\#openAccessPolicy). 


\section{Introduction}

Burn and fire injuries are among public health concerns worldwide, especially in low-and-middle-income countries where fire prevention remains substandard. In 2014, statistics from the United States (US) showed that death from fire and burn injuries occurred to 3,194 people or at an estimated incidence of $1 / 100,000$ population. Thermal injuries represent $1.3 \%$ of all injuries and the problem accounts for $1.6 \%$ of all trauma-related fatalities. When the mortality figures from burn injuries were analyzed with the 408,945 non-fatal burns reported in the US in the same year, burn-related deaths were about $0.8 \%$. According to the American Burn Association, approximately 40,000 hospitalizations in the US in the year 2016 was related to burn conditions and, among these, 3/4s were admitted to a burn center. In addition to the preceding data, the trend line reported by the web-based injury statistics query and reporting system governed by the US Centers for Disease Control and Prevention showed that the total number of burn patients was decreasing in general, but increasing in elderly population. ${ }^{2}$ Considering the global incidence of fire and burn injuries, South East Asia was the area with the highest mortality rate from flame burn injuries with an incidence of 11.6 per 100,000 population. ${ }^{3}$

There are various causes of burn injury in patients admitted to burn centers. Flame burn is the predominant cause, followed by scald burn, heat contact, and electrical and chemical burns. Although the majority of incidences occur at home, burn injuries can also occur at work or on the street. A study from Iran reported that $60.0 \%$ of burns were accidental while most of the rest was occupational injuries. ${ }^{4}$ The age groups at high risk for burn injury are children under four years of age and the elderly more than 60 years of age. Management of severe burns requires intensive care from the scene to the burn center and usually involves high-cost treatment. It costs about
US $\$ 1,000$ per patient per day to provide adequate burn care in the developed world. Nevertheless, mortality in burn patients in these resource-competent countries was reported as high as $3.3 \%{ }^{5}$

In resource-limited countries, the mortality of severe burn patients is very high. A study from Iran reported an overall probability of death of $26.0 \%{ }^{4}$ while a study from Pakistan reported $41.0 \%$ mortality. ${ }^{3}$ It has been estimated that more than $90.0 \%$ of burn-related deaths currently occur in low and middle income countries. ${ }^{6}$ The depth and extent of the burn are the most crucial factors associated with mortality. However, death can occur in severe burn patients not only from the acute physiologic insults caused by the burn itself, but may occur later during the admission due to hospital-acquired infections such as pneumonia or wound infection. Additionally we can use clinical and laboratory factors to predict mortality in burn patients and adjust our treatment plan accordingly. Suicidal attempt, female gender, extreme $\mathrm{age}^{7}$, and also co-morbidities and complications have been associated with increased mortality risk. ${ }^{5}$

Songklanagarind Hospital is a level-1 trauma center with a burn center situated in the southern part of Thailand. The burn center receives approximately 3-5 severe burn patients per month from the surrounding hospitals. A rough figure of in-hospital mortality of burn patients in our center is $10.0 \%$. In this study, we aimed to examine the characteristics of severe burn patients in our service, beginning with quantitation of the exact number of inhospital mortalities, and we also analyzed the factors associated with in-hospital mortality in these cases. The causes of injury, intensive care requirement, ventilator days, length of hospital stay, in-hospital expenditures and functional status after discharge were studied. It was expected that findings from the study would be useful in improving our care of burn patients. 


\section{Material and Methods}

The study was approved by the Human Research Ethics Committee of the Faculty of Medicine, Prince of Songkla University (REC number 62-329-10-1). A retrospective study was conducted in the Department of Surgery at Songklanagarind Hospital. The study included major burn patients who were admitted to the burn unit or the intensive care unit between the years 2011 and 2018. Data collection began on the first day of admission and during the hospital course until discharge or death. Demographic data, admission laboratories, total body surface area injured from the burn, area or areas of injury, length of stay, wound dressing material, and nutrition supplements were recorded. The total burn surface area (TBSA) in terms of $\%$ total body surface area was calculated from the Lune and Browder chart. ${ }^{1}$ A severe burn was defined as TBSA $>20.0 \%$. More than $90.0 \%$ of the severe burn patients were initially admitted to the burn unit. Fluid resuscitation used a modified Parkland formula protocol, with a central venous catheter placed in either the subclavian or the femoral vein during the initial resuscitation phase. Microbiological studies including blood cultures or burn wound cultures were performed when there were clinical indications. In-hospital mortality was defined as death occurring during the admission, regardless of the cause of death. In order to analyze for factors associated with survival, parameters from the survival group were compared with those of the nonsurvivor group.

All values are expressed as median and standard deviation or as percentages, with each variable tested for differences between the survivor and non-survivor groups by univariate statistical methods (chi-squared test or Fisher's exact test) with significance accepted at $p$-value $<0.05$, and all significant variables by univariate analysis then included in multivariate analysis. Stepwise logisticregression analysis was performed with selected predictive factors for death. This analysis resulted in the final predictive model.

A nomogram was then constructed from the three statistically significant factors using the method proposed by Zang et al. $^{8}$ The data set was separated into two parts at the proportion of $3: 7.30 .0 \%$ of the data was used to perform an internal validation of the nomogram. The nomogram's sensitivity, specificity, positive predictive value, negative predictive value and accuracy were calculated for death at various cut-off points of the total scores. The highest performance nomogram with optimal cut-off points was selected based on a Receiver Operating characteristic curve $(\mathrm{ROC})$ and the area under the ROC was plotted. For interpretation of the ROC, area under the curve (AUC) values of $\geq 0.90$ "excellent," $\geq 0.80$ "good," $\geq 0.70$ "fair," and $<0.70$ "poor" were used. The statistical analysis was performed using $R$ version 3.4.0 software (R Foundation, Vienna, Austria).

\section{Results}

Of 245 major burn patients admitted to our hospital during the study period, 127 were classified as severe burns and were included in this study. The baseline characteristics of the 127 severe burn patients are presented in Table 1. Eighty-eight patients were male, the mean age of the 127 patients was 33 years (range 21-43 years) and the mean TBSA was $42.5 \%$ (range 25.5-53.0\%). Eighty patients $(63.0 \%)$ had flame injury, 22 patients $(17.3 \%)$ had electrical injury, 16 patients (12.6\%) had scald injury and 9 patients $(7.1 \%)$ had chemical injury. The mean length of stay was 68 days (range 13-86 days). Twenty-four patients (19.0\%) died during their stay in the hospital and the median time to death in this group was 14.5 days (range 6-53.2 days). All patients with severe infection had positive blood culture. The most common pathogens in both groups were Pseudomonas aeruginosa and Acinetobacter baumannii. We also found drug-resistant 
Table 1 Demographic and clinical data of study severe burn patients

\begin{tabular}{|c|c|c|c|}
\hline Parameter & Frequency (\%) & Median & Percentile 25-Percentile 75 \\
\hline \multicolumn{4}{|l|}{ Sex } \\
\hline Male & $88(69.3)$ & & \\
\hline Female & $39(30.7)$ & & \\
\hline Age (years) & & 31 & $21.00-43.00$ \\
\hline $0-32$ & $59(46.5)$ & & \\
\hline$>32$ & 68 (53.5) & & \\
\hline Weight $(\mathrm{kg})$ & & 60 & $55.00-65.00$ \\
\hline Body mass index $\left(\mathrm{kg} / \mathrm{m}^{2}\right)$ & & 22.2 & $18.63-25.63$ \\
\hline \multicolumn{4}{|l|}{ Underlying disease } \\
\hline Diabetes mellitus & $2(1.6)$ & & \\
\hline Hypertension & $7(5.5)$ & & \\
\hline Dyslipidemia & $4(3.2)$ & & \\
\hline Others & $5(3.9)$ & & \\
\hline \multicolumn{4}{|l|}{ Associated injury } \\
\hline Head injury & $7(5.5)$ & & \\
\hline Maxillofacial injury & $1(0.8)$ & & \\
\hline Torso injury & $4(3.2)$ & & \\
\hline Limb fracture & $2(1.6)$ & & \\
\hline \multicolumn{4}{|l|}{ Initial laboratory values } \\
\hline Hemoglobin $(\mathrm{g} / \mathrm{dL})$ & & 14.1 & $12.00-16.30$ \\
\hline Leukocytes (cells $/ \mathrm{mm}^{3}$ ) & & 12,080 & $8,475-19,162$ \\
\hline Platelets ( $10^{\wedge} 3$ cells/cu.mm.) & & 227.5 & $193.00-357.00$ \\
\hline Creatinine & & 0.91 & $0.64-1.08$ \\
\hline Serum albumin $(\mathrm{g} / \mathrm{dL})$ & & 2.65 & $2.00-3.20$ \\
\hline Burn area (\%TBSA) & & 40 & $25.50-53.00$ \\
\hline \multicolumn{4}{|l|}{ Number of operations (times) } \\
\hline $0-3$ & $37(31.4)$ & & \\
\hline $4-5$ & $81(68.6)$ & & \\
\hline$>5$ & $9(7.1)$ & & \\
\hline Time to first skin graft & & 34 & $21.00-57.00$ \\
\hline Length of hospital stay (days) & & 45 & $13.00-86.50$ \\
\hline
\end{tabular}

$\mathrm{kg} / \mathrm{m}^{2}=$ kilogram per square meter, $\mathrm{g} / \mathrm{dL}=$ gram per deciliter, $\mathrm{mm}^{3}=$ cubic millimeter, cu.mm.=microliter, TBSA=total body surface area

microbes, most commonly methicillin-resistant Staphylococcus aureus. In cases with infection, the major causes of death were burn wound sepsis and ventilator-associated pneumonia. The main causes of death in patients without infection were pulmonary- and cardiac-related causes.

A comparison between the survival and nonsurvival groups is shown in Table 2. On univariate analysis, factors associated with in-hospital mortality included age more than 32 years, TBSA more than $55.0 \%$, facial involvement, hemoglobin of less than $9 \mathrm{~g} / \mathrm{dL}$, creatinine of more than $1.2 \mathrm{mg} / \mathrm{dL}$, delayed feeding initiation and receiving blood transfusion (Table 3 ). Stepwise logistic regression showed three factors independently associated with mortality, including TBSA, high creatinine and facial involvement (Table 4). 
Table 2 Comparisons between survival and mortality groups

\begin{tabular}{|c|c|c|c|c|}
\hline Parameter & Number (\%) & Survival cases & Mortality cases & $p$-value \\
\hline All & $127(100.0)$ & $103(81.1)$ & $24(18.9)$ & \\
\hline \multicolumn{5}{|l|}{ Age (years) } \\
\hline $0-32$ & $59(46.4)$ & 57 (55.3) & $2(8.3)$ & \multirow[t]{2}{*}{$<0.001$} \\
\hline$>32$ & $68(53.5)$ & $46(44.7)$ & $22(91.7)$ & \\
\hline \multicolumn{5}{|l|}{ Sex } \\
\hline Female & $39(30.7)$ & $29(28.2)$ & $10(41.7)$ & \multirow[t]{2}{*}{0.300} \\
\hline Male & $88(62.3)$ & $74(71.8)$ & $14(58.3)$ & \\
\hline \multicolumn{5}{|c|}{ Body mass index $\left(\mathrm{kg} / \mathrm{m}^{2}\right)$} \\
\hline$<23.9$ & $30(60.0)$ & $26(63.4)$ & $4(44.4)$ & \multirow[t]{2}{*}{$0.450^{*}$} \\
\hline$>23.9$ & $20(40.0)$ & $15(36.6)$ & $5(55.6)$ & \\
\hline \multicolumn{5}{|c|}{ Associated injury } \\
\hline Present & $13(10.2)$ & $10(9.7)$ & $3(12.5)$ & \multirow[t]{2}{*}{$0.710^{*}$} \\
\hline Absent & $114(89.8)$ & $93(90.3)$ & $21(87.5)$ & \\
\hline \multicolumn{5}{|c|}{ Burn area (\%TBSA) } \\
\hline $0.0-55.0$ & $103(81.1)$ & $91(88.3)$ & $6(25.0)$ & \multirow[t]{2}{*}{$<0.010$} \\
\hline$>55.0$ & $24(18.9)$ & $12(11.7)$ & $18(75.0)$ & \\
\hline \multicolumn{5}{|l|}{ Burn site** } \\
\hline Face & $58(45.7)$ & 37 (35.9) & $21(87.5)$ & $<0.010^{*}$ \\
\hline Trunk & $96(75.6)$ & $74(71.8)$ & $22(97.1)$ & 0.076 \\
\hline Extremities & $120(94.5)$ & $97(94.2)$ & $23(95.8)$ & 1.000 \\
\hline \multicolumn{5}{|c|}{ Hemoglobin $(\mathrm{g} / \mathrm{dL})$} \\
\hline $0-9$ & $12(9.5)$ & $7(6.8)$ & $5(21.7)$ & \multirow[t]{2}{*}{0.040} \\
\hline$>9$ & $114(90.5)$ & 96 (93.2) & $18(78.3)$ & \\
\hline \multicolumn{5}{|c|}{ Serum creatinine (mg/dL) } \\
\hline $0-1.2$ & $106(84.8)$ & $92(90.2)$ & $14(60.9)$ & \multirow[t]{2}{*}{0.020} \\
\hline$>1.2$ & $19(15.2)$ & $10(9.8)$ & $9(39.1)$ & \\
\hline \multicolumn{5}{|c|}{ Serum albumin ( $\mathrm{g} / \mathrm{dL})$} \\
\hline $0-3.5$ & $84(80.8)$ & $65(80.2)$ & $19(82.6)$ & \multirow[t]{2}{*}{1.000} \\
\hline$>3.5$ & $20(19.2)$ & $16(19.8)$ & $4(17.4)$ & \\
\hline \multicolumn{5}{|l|}{ Escharotomy } \\
\hline No & $114(89.8)$ & 95 (92.2) & $19(79.2)$ & \multirow[t]{2}{*}{$0.070^{*}$} \\
\hline Yes & $13(10.2)$ & $8(7.8)$ & $5(20.8)$ & \\
\hline \multicolumn{5}{|l|}{ Early feeding } \\
\hline No & $10(7.9)$ & $4(3.9)$ & $6(25)$ & \multirow[t]{2}{*}{$<0.003^{*}$} \\
\hline Yes & $117(92.1)$ & $99(96.1)$ & $18(75)$ & \\
\hline \multicolumn{5}{|c|}{ Blood transfusion } \\
\hline No & $64(50.4)$ & $57(55.3)$ & 7 (29.2) & \multirow[t]{2}{*}{0.037} \\
\hline Yes & $63(49.6)$ & $46(44.7)$ & $17(70.8)$ & \\
\hline
\end{tabular}

*Fisher's exact test, **Some patients had more than 1 sites

$\mathrm{kg} / \mathrm{m}^{2}=\mathrm{kilogram}$ per square meter, TBSA=total body surface area, $\mathrm{g} / \mathrm{dL}=$ gram per deciliter, $\mathrm{mg} / \mathrm{dL}=$ milligram per deciliter 
Table 3 Univariate logistic regression analysis

\begin{tabular}{lll}
\hline Parameter & Odds ratio & 95\% Cl \\
\hline Age $>32$ years & 13.63 & $3.05-61.01$ \\
Burn area $>55.0 \%$ TBSA & 22.75 & $7.55-68.53$ \\
Face involvement & 12.49 & $3.49-44.68$ \\
Trunk involvement & 4.31 & $0.95-19.51$ \\
Hemoglobin $<9 \mathrm{~g} / \mathrm{dL}$ & 3.81 & $1.09-13.34$ \\
Creatinine $>1.2 \mathrm{mg} / \mathrm{dL}$ & 5.91 & $2.05-17.10$ \\
Early feeding & 8.25 & $2.11-32.18$ \\
Blood transfusion & 3.01 & $1.15-7.88$ \\
\hline
\end{tabular}

$\mathrm{Cl}=$ confidence interval, TBSA=total body surface area, $\mathrm{mg} / \mathrm{dL}=$ milligram per deciliter, $\mathrm{g} / \mathrm{dL}=\mathrm{gram}$ per deciliter

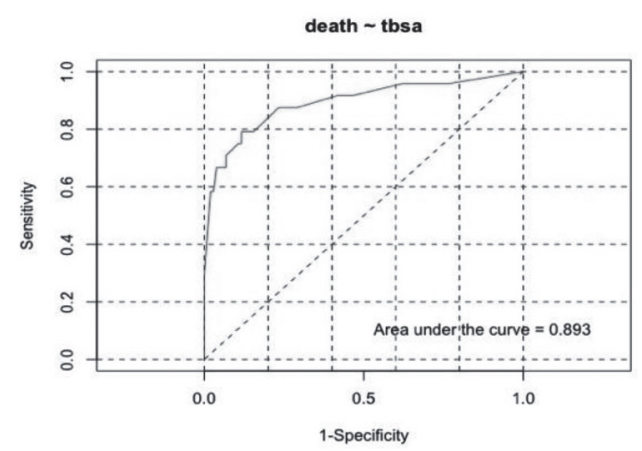

A

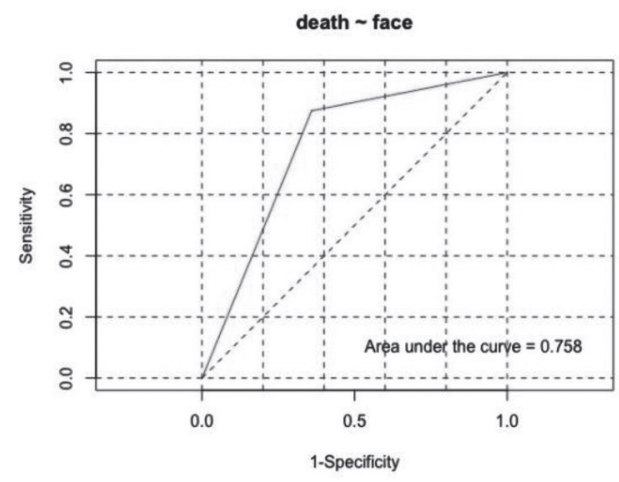

C
Table 4 Stepwise logistic regression (final model)

\begin{tabular}{lll}
\hline Parameter & Odds ratio & $95 \% \mathbf{C l}$ \\
\hline Burn area $>55.0 \% \mathrm{TBSA}$ & 10.28 & $1.00-105.35$ \\
Creatinine $>1.2 \mathrm{mg} / \mathrm{dL}$ & 13.53 & $1.20-152.03$ \\
Face involvement & 15.28 & $1.23-190.14$ \\
\hline
\end{tabular}

$\mathrm{Cl}=$ confidence interval, TBSA=total body surface area, $\mathrm{mg} / \mathrm{dL}=$ milligram per deciliter

On an ROC curve plot between TBSA and probability of mortality, the AUC was 0.89 (Figure 1A). When we used a TBSA cur-off of $55.0 \%$, the sensitivity was $11.6 \%$ and the specificity $75.0 \%$, with a calculated lethal area

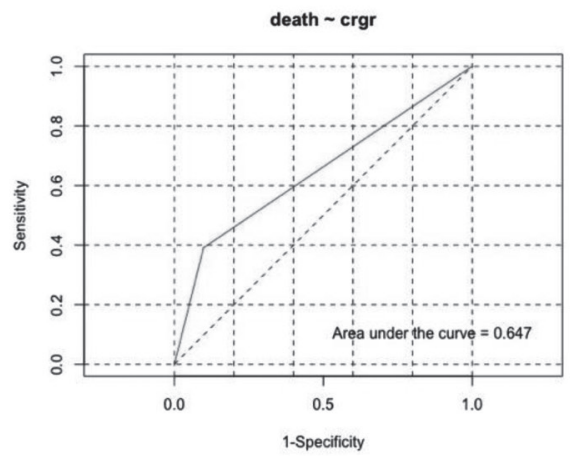

$\mathbf{B}$

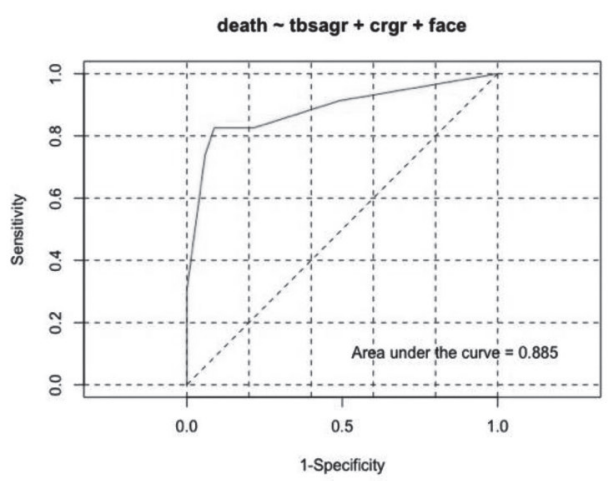

D

Figure 1 The receiver operating characteristic curve plots of burn area, blood creatinine, face involvement and probability of mortality 
fifty percent (LA50) of $76.0 \%$ of TBSA. Serum creatinine had prediction performance in the AUC of 0.647 (Figure 1B). Patients who had blood creatinine more than $1.2 \mathrm{mg} / \mathrm{dL}$ had increased mortality with sensitivity of $39.0 \%$ and specificity of $98.0 \%$. Patients with face involvement had increased mortality with sensitivity of $35.9 \%$ and specificity of $87.5 \%$, with an AUC of 0.76 (Figure $1 \mathrm{C}$ ). On combining together the three significant factors, the AUC was 0.89 (Figure 1D).
The three significant factors were used to construct a nomogram with an aim to enable the physician to predict mortality (Figure 3 ). This nomogram predicted the probability of death using a TBSA $>55.0 \%$, face involvement and blood creatinine more than $1.2 \mathrm{mg} / \mathrm{dL}$. Using a cutoff score of 114 , this nomogram scoring system had an ROC-AUC of 0.84 .

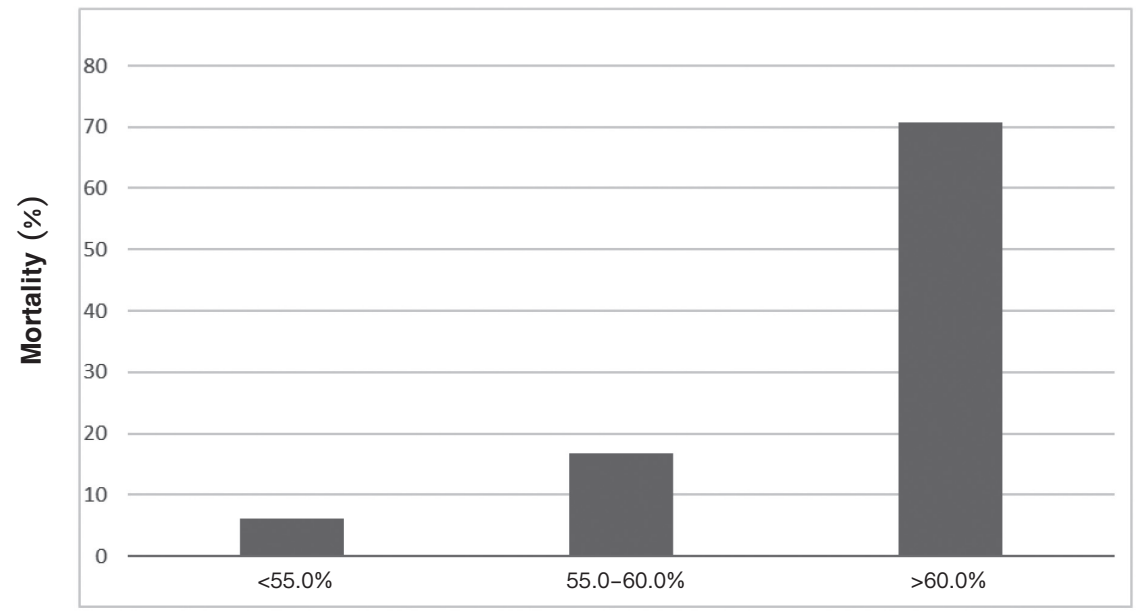

Burn area (\%)

Figure 2 Bar chart showing the association between mortality and extent of burn area

Points
TBSA
Cr_1.2
Total Points
Death

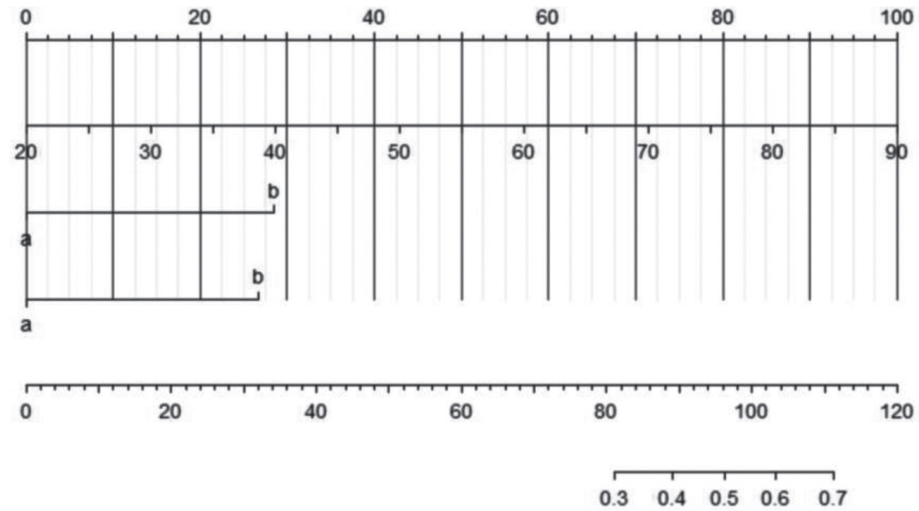

TBSA=total body surface area

Figure 3 Nomogram to predict mortality in severe burn patients 


\section{Discussion}

Patients with severe burn injury have a high chance of in-hospital mortality due to various reasons, both direct thermal injuries to vital organs and complications occurring during the treatment process. Some of these complications are preventable by improving the care process. A recent study from the Netherlands found a reduction in major burn mortality from $23.0 \%$ during the years $1987-1996$ to $7.0 \%$ during the years $2007-2016$. $^{910}$ A study from Canada in which $15.0 \%$ overall mortality was reported emphasized that multi-organ failure and infectious complications were the two most common causes of death in major burn patients. ${ }^{9}$ The mortality rate of $20.0 \%$ in our severe burn patients in 10 years and it was higher than reports from developed countries. ${ }^{11}$ However, our figure was comparable with other reports from developing countries, where flame burn remains a cause of major trauma-related fatalities. ${ }^{3,12,13}$ Consistent with all previous studies, TBSA was the most influential factor determining outcome in burn patients. ${ }^{14,15}$ Our study also found that the average LA50 of our patients was $76.0 \%$ of TBSA, which was comparable with other studies from southern Iran which found the LA50 was $66.5 \%$ of TBSA. ${ }^{16,17}$

Effective initial resuscitation, including airway management and circulatory system restoration, is the crucial factor for survival in burn patients. Among the 24 mortality cases in our series, $5(20.0 \%)$ occurred within five days and 12 (50.0\%) occurred within 14 days of admis-sion. All 12 cases with mortality within the first 14 days had TBSA $>40.0 \%$ and 11 cases had facial involvement. Taken together with our finding that high serum creatinine was associated with unfavorable outcomes, the evidence suggests that management of circulatory failure and special attention to inhalation injuries should be priorities in improving the care process. As burns involving the facial area are associated with higher risk of inhalation injury ${ }^{18-20}$, airway and respiratory care should be promptly provided in patients with facial burns no matter how extensive the TBSA is. ${ }^{21,22}$ Early bronchoscopy in order to grade the severity of inhalation injury is advisable for cases with suggestive evidence such as signs of airway edema, respiratory failure, carboxyhemoglobinemia and adult respiratory distress syndrome. An association between high serum creatinine on arrival and in-hospital mortality in our series indicates that hypoperfusion is also an issue that needs further attention. Early provision of fluid resuscitation has been advocated as the most pertinent care in the initial phase of burn management. ${ }^{1}$ Although prehospital care is available in major cities in Thailand, secure venous access and large volume resuscitation are frequently not successful, especially in cases with extensive burns involving one or more extremities. The issues of under-resuscitation and inhalation injury have also been addressed in burn case series from other south Asian countries. ${ }^{3,23}$ Future research should focus on how and how much fluid should be initiated at accident scenes and during transportation to the emergency department.

On univariate analysis, our study found that factors other than TBSA, facial involvement and high serum creatinine were associated with in-hospital mortality including age, anemia, and requirement of blood transfusion and delayed enteral feeding. Although these factors were statistically regarded as co-variate on multivariate analysis, they should not be overlooked. Anemia in burn patients can be a result of continuing blood loss from wounds and poorer blood cell production secondary to nutritional deprivation and septicemia. Early enteral feeding has been suggested by recent studies as a strategy which might ameliorate gut bacterial translocation and sepsis. ${ }^{11}$ Elderly age has also been reported by various studies to be a determinant of adverse outcomes. ${ }^{12}$ In our study, we found the cutoff age that gave different survival outcomes was around 35 years. This might be explained by the fact 
that we seldom have burn cases in the elderly. Another explanation could be that young patients have more physiologic reserves and fare better through the treatment process.

Concerning the three factors independently associated with adverse outcomes in our patients, TBSA, facial involvement and high serum creatinine on admission, we found that a nomogram constructed based on the combination of these three factors had good performance in predicting in-hospital mortality in our patients. This nomogram could help in the future in calculating survival probability using these three parameters as inputs. The aim of using this nomogram is to quickly categorize burn patients into those with high risk and thus requiring more intensive resuscitation and invasive monitoring. In our setting, such high-risk patients should be immediately taken to the intensive care unit and provided with intensive physiologic support.

\section{Conclusion}

This study reviewed survival outcomes of severe burn patients in a tertiary setting in southern Thailand and found that the extent of injury, facial involvement and azotemia on admission were three factors independently related to in-hospital mortality. These parameters were used to construct a nomogram that predicts high-risk cases.

\section{Conflicts of interest}

All authors declare no personal or professional conflicts of interest

\section{References}

1. David N, Rajeev B Ahuja, J F Aili Low, Brett D. Arnoldo, Amina EL Ayadi, et al. Total burn care. $5^{\text {th }}$ ed. New York: Elsevier; 2018.

2. Fatal Injury Data WISQARS Injury Center CDC [homepage on the Internet]. Atlanta: CDC; 2019 [cited 2019 Sep 17]. Available from: https://www.cdc.gov/injury/wisqars/fatal.htm
3. Siddiqui E, Zia N, Feroze A, Awan S, Ali AL, Razzak JA, et al. Burn injury characteristics: findings from the Pakistan National Emergency Department Surveillance Study. BMC Emerg Med 2015;15:5

4. Naghavi M, Abolhassani F, Pourmalek F, Moradi Lakeh M, Jafari N, Vaseghi $S$, et al. The burden of disease and injury in Iran 2003. Popul Health Metr 2009;7:9.

5. Zavlin D, Chegireddy V, Boukovalas S, Nia AM, Branski LK, Friedman JD, et al. Multi-institutional analysis of independent predictors for burn mortality in the United States. Burns Trauma 2018;6:24.

6. Forjuoh SN. Burns in low- and middle-income countries: a review of available literature on descriptive epidemiology, risk factors, treatment, and prevention. Burns $\mathrm{J}$ Int Soc Burn Inj 2006;32:529-37.

7. Fazeli S, Karami-Matin R, Kakaei N, Pourghorban S, SafariFaramani R, Safari-Faramani B. Predictive factors of mortality in burn patients. Trauma Mon 2014;19. doi: 10.5812 traumamon.14480.

8. Zhang Z, Kattan MW. Drawing Nomograms with R: applications to categorical outcome and survival data. Ann Transl Med 2017;5:211.

9. Dokter J, Vloemans AF, Beerthuizen GIJM, van der Vlies $\mathrm{CH}$, Boxma $\mathrm{H}$, Breederveld $\mathrm{R}$, et al. Epidemiology and trends in severe burns in the Netherlands. Burns 2014;40:1406-14.

10. Gigengack RK, van Baar ME, Cleffken BI, Dokter J, van der Vlies $\mathrm{CH}$. Burn intensive care treatment over the last 30 years: Improved survival and shift in case-mix. Burns 2019; 45:1057-65.

11. Jeschke MG, Pinto R, Kraft R, Nathens AB, Finnerty CC, Gamelli RL, et al. Morbidity and survival probability in burn patients in modern burn care. Crit Care Med 2015;43:808-15.

12. Khan N, Malik MAN. Presentation of burn Injuries and their management outcome. J Pak Med Assoc 2006;56:394-7.

13. Tripathee S, Basnet SJ. Epidemiology of burn injuries in Nepal: a systemic review. Burns Trauma 2017;5:10.

14. Kobayashi K, Ikeda H, Higuchi R, Nozaki M, Yamamoto $Y$, Urabe $\mathrm{M}$, et al. Epidemiological and outcome characteristics of major burns in Tokyo. Burns 2005;31:3-11.

15. Tian H, Wang L, Xie W, Shen C, Guo G, Liu J, et al. Epidemiologic and clinical characteristics of severe burn patients: results of a retrospective multicenter study in China, 2011-2015. Burns Trauma 2018;6:14. 
16. Steinvall I, Elmasry M, Fredrikson M, Sjoberg F. Standardised mortality ratio based on the sum of age and percentage total body surface area burned is an adequate quality indicator in burn care: an exploratory review. Burns 2016;42: 28-40.

17. Keshavarzi A, Kardeh S, Pourdavood A, Mohamadpour M, Dehghankhalili M. Determinants of the lethal area 50 index (LA50) in burn patients admitted to a tertiary referral burn center in Southern Iran. Bull Emerg Trauma 2018;6:59-63.

18. Foncerrada G, Culnan DM, Capek KD, González-Trejo S, Cambiaso-Daniel J, Woodson LC, et al. Inhalation injury in the burned patient. Ann Plast Surg 2018;80:98-105.

19. Karki D, Muthukumar V, Jatin B. Concept of lethal triad in critical care of severe burn injury. Indian J Crit Care Med 2019;23:206-9.

20. Iyun AO, Ademola SA, Olawoye O, Michael Al, Oluwatosin OM. Comparative review of burns with inhalation injury in a tertiary hospital in a developing country wound. Wound 2016;28:1-6.

21. Dries DJ, Endorf FW. Inhalation injury: epidemiology, pathology, treatment strategies. Scand J Trauma Resusc Emerg Med 2013;21:31.

22. Berger MM. Nutrition determines outcome after severe burns. Ann Transl Med 2019;7:216.

23. McGill V. Outcome for older burn patients. Arch Surg 2000;135: 320. 